\title{
Evaluation of haematological and serum biochemical profile of propofol induced isoflurane anaesthesia in geriatric dogs premedicated with diazepam and butorphanol
}

\author{
(iD) \\ (iD)
}

M.R. Manasa1', K.M. Dileep kumar², S. Anoop³ ${ }^{3}$ Soumya Ramankutty ${ }^{4}$, V. Beena ${ }^{5}$ and K.D. John Martin ${ }^{6}$ Department of Veterinary Surgery and Radiology, College of Veterinary and Animal Sciences, Mannuthy, Thrissur, Kerala - 680 651. Kerala Veterinary and Animal Sciences University, India.

Citation: Manasa, M.R., Dileepkumar, K.M., Anoop, S., Ramankutty, S., Beena, V. and John Martin, K.D. 2021. Evaluation of haematological and serum biochemical profile of propofol induced isofluraneanaesthesia in geriatric dogs premedicated with diazepam and butorphanol. J. Vet. Anim. Sci. 52(1): 81-84. DOI: https://doi.org/10.51966/jvas.2021.52.1.81-84

Received : 30.11 .2020

Accepted: 13.12.2020

Published: 01.01.2021

Abstract

Six geriatric dogs of various breeds belonging to either sex presented for various surgical procedures to Teaching Veterinary Clinical Complex, Mannuthy and University Veterinary Hospital, Kokkalai, were selected for the study. On the day of surgery, preanaesthesia was performed with diazepam and butorphanol. After ten minutes of premedication, preoxygenation was carried out for three minutes. Propofol (1 per cent w/V) was administered as a slow bolus, intravenous injection for induction of anaesthesia. Maintenance of surgical plane of anaesthesia was carried out with isoflurane in oxygen using Bain's circuit system incorporated with isoflurane vapourizer. Haematological and serum biochemical parameters, which were recorded before premedication, ten minutes after commencement of isoflurane and after recovery from general anaesthesia are described here. The present study concluded that the anaesthetic protocol is safe in geriatric animals with regard to haematological and serum biochemical parameters.

Keywords: geriatric dogs, anaesthesia, haematological and serum parameters

Geriatric patients are defined as those individuals that have completed 75 to 80 per cent of their expected life span (Baetge and Matthews, 2012). According to Joubert (2007) it is necessary to evaluate the geriatric patient before anaesthesia to know the underlying disease conditions. Hence the drugs used for anaesthesia in these animals must be titrated in their dose

Corresponding Author-email- anoop@kvasu.ac.in Ph :9446792333

1. MVSc Scholar - email - manasadr07@gmail.com

2. Veterinary Surgeon, Animal Husbandry Department, Government of Kerala.

3. Associate Professor

4. Assistant Professor

5. Assistant Professor, Department of Veterinary Physiology

6. Professor and Head

Copyright: () 2021 Manasa et al. This is an open access article distributed under the terms of the Creative Commons Attribution 4.0 International License (http://creativecommons.org/licenses/by/4.0/), which permits unrestricted use, distribution, and reproduction in any medium, provided the original author and source are credited. 
and administered according to the health of the animal. This paper places on record of haematological and serum biochemical profile of propofol induced isoflurane anaesthesia in geriatric dogs premedicated with diazepam and butorphanol.

\section{Materials and Methods}

Six geriatric dogs aged from ten to nineteen years of various breeds of either sex presented to the University Veterinary Hospitals, at Mannuthy and Kokkalai of Kerala Veterinary and Animal Sciences University for various surgical procedures were selected for the study. All the animals were subjected for thorough preanaesthetic evaluation before surgery. Food was withheld for 12 hours and water for six hours before administration of the anaesthesia. Diazepam at the dose rate of $0.25 \mathrm{mg}$ per $\mathrm{kg}$ body weight and butorphanol at the dose rate of $0.2 \mathrm{mg}$ per $\mathrm{kg}$ body weight were administered intravenously as pre-anaesthetic medication at one minute interval. After ten minutes of premedication, pre-oxygenation was carried out in all animals for three minutes. After pre-oxygenation, propofol ( $1 \% \mathrm{w} / \mathrm{v})$ emulsion was administered as slow intravenous bolus injection, to effect general anaesthesia. Soon after induction animals were intubated with appropriate sized endotracheal tube. Anaesthesia was maintained with isoflurane in oxygen at the rate of $100 \mathrm{~mL} / \mathrm{kg}$ body weight, by using Bain's circuit system incorporated with isoflurane vapourizer.

All the vital parameters were monitored during preanaesthesia, maintenance and recoveryperiod.Bloodsampleforhaematological and serum biochemical studies was collected and analysed before premedication, ten minutes after commencement of isoflurane administration and after recovery from general anaesthesia. The parameters like rectal temperature, pulse rate, respiration rate, heart rate, capillary refilling time and colour of mucus membrane were observed after administration of anaesthesia in every five minutes were recorded. The animals were prepared for the relevant surgical procedures, under strict aseptic conditions and surgery was performed as per standard procedures.
Amoxicillin at the dose rate of 10 mg per kg body weight IV and meloxicam at the dose rate of $0.2 \mathrm{mg}$ per $\mathrm{kg}$ body weight IM was administered before the surgery to all the animals. Postoperatively, animals were maintained with antibiotic and anti-inflammatory drugs orally for five consecutive days.

\section{Results and Discussion}

The mean age of geriatric dogs selected for the study was $13.33 \pm 1.33$ years and the mean body weight was $15.8 \pm 2.40 \mathrm{~kg}$. The mean values of the haematological and biochemical parameters are expressed in Table 1 and 2 .

The mean value of total erythrocyte count and the mean total leucocyte count decreased after ten minutes of isoflurane commencement with non-significant and significant changes respectively and returned to baseline values after recovery from surgical plane of general anaesthesia with significantand non-significant changes respectively. Similar results were obtained with propofol-isoflurane anaesthesia in dogs by Kavechiya (2010). The reason for decreased total erythrocyte count might be the pooling of red blood cells in the spleen because of stimulated adrenocortical area and the interstitial fluid migrating in to the circulating compartment (Sankar et al., 2011). Isoflurane is more immunosuppressive compared to propofol and thus might have resulted in the decreased leucocyte count due to changes in the anti-inflammatory cytokines during trans-anaesthetic period (Tomihari et al., 2015).

The meanlymphocyte count increased non-significantly while mean monocytes per cent increased significantly after ten minutes of isoflurane commencement which returned to baseline values after recovery from anaesthesia without any significant change. The mean granulocytes per cent decreased after ten minutes of isoflurane commencement which returned to baseline values after recovery from anaesthesia without any significant change. The results are in accordance with the observations made by Kavechiya (2010), Ramankutty (2008) and Zlateva and Marinov (2015) respectively 
Table 1. Observations on haematological parameters (Mean \pm SE) $n=6$

\begin{tabular}{|l|l|l|l|}
\hline \multicolumn{1}{|c|}{ Parameters } & Before premedication & $\begin{array}{c}\text { Ten minutes after } \\
\text { commencement } \\
\text { of Isoflurane } \\
\text { administration }\end{array}$ & $\begin{array}{c}\text { After recovery } \\
\text { from general } \\
\text { anaesthesia. }\end{array}$ \\
\hline Total erythrocyte count $\left(10^{6} / \mu \mathrm{L}\right)$ & $4.62 \pm 0.2$ & $3.47 \pm 0.22$ & $4.59 \pm 0.36^{*}$ \\
\hline Total leucocyte count $\left(10^{3} / \mu \mathrm{L}\right)$ & $14.92 \pm 2.04$ & $10.07 \pm 1.84^{*}$ & $12.97 \pm 2.07$ \\
\hline Lymphocyte $(\%)$ & $22.5 \pm 2.63$ & $26.93 \pm 5.69$ & $24.57 \pm 3.27$ \\
\hline Monocytes $(\%)$ & $6.27 \pm 0.4$ & $7.53 \pm 0.42^{*}$ & $6.92 \pm 0.57$ \\
\hline Granulocytes $(\%)$ & $71.23 \pm 2.84$ & $65.62 \pm 5.95$ & $69.52 \pm 3.38$ \\
\hline Haemoglobin $(\mathrm{g} / \mathrm{dL})$ & $9.83 \pm 1.20$ & $7.78 \pm 0.91$ & $9.57 \pm 1.3$ \\
\hline Volume of packed red cells $(\%)$ & $26.27 \pm 3.17$ & $20.52 \pm 1.78$ & $23.77 \pm 3.27$ \\
\hline
\end{tabular}

Means with "*' as superscript within a row differ significantly at $5 \%$ significance level

Table 2. Observations on serum biochemistry profile (Mean \pm SE) $n=6$

\begin{tabular}{|l|l|l|l|}
\hline \multicolumn{1}{|c|}{ Parameters } & Before premedication & $\begin{array}{c}\text { Ten minutes after } \\
\text { commencement } \\
\text { of Isoflurane } \\
\text { administration }\end{array}$ & $\begin{array}{c}\text { After recovery } \\
\text { from general } \\
\text { anaesthesia. }\end{array}$ \\
\hline Alanine aminotransferase (IU/L) & $46.26 \pm 10.9$ & $39.43 \pm 7.76$ & $33.42 \pm 6.88$ \\
\hline Aspartate aminotransferase (IU/L) & $32.28 \pm 6.07$ & $26.3 \pm 2.15$ & $32.29 \pm 4.0$ \\
\hline Blood Urea Nitrogen $(\mathrm{mg} / \mathrm{dL})$ & $13.59 \pm 3.34$ & $9.07 \pm 1.17$ & $8.1 \pm 1.34$ \\
\hline Creatinine $(\mathrm{mg} / \mathrm{dL})$ & $1.11 \pm 0.15$ & $1.01 \pm 0.03$ & $1.01 \pm 0.04$ \\
\hline
\end{tabular}

Means with "*' as superscript within a row differ significantly at $5 \%$ significance level

for changes of lymphocyte, monocyte and granulocyte per cent during propofol-isoflurane anaesthesia. The reason could be stimulation of lymphocytes and neutrophils by glucocorticoids due to stimulation at adrenocortical region during general anaesthesia (Brand et al., 2003).

The mean haemoglobin, volume of packed red cells, alanine aminotransferase, aspartate aminotransferase, blood urea nitrogen and creatinine decreased after ten minutes of isoflurane commencement and returned to baseline values after recovery from anaesthesia without any significance. The results are in agreement with the observations made by Sen and Kilic (2018) with propofolisoflurane anaesthesia in geriatric dogs. According to Sankar et al. (2011) the reason for the decrease in haemoglobin concentration could be due to pooling of erythrocytes in to the spleen. Decreased total erythrocyte count might have also attributed to the decrease in the haemoglobin level during general anaesthesia During general anaesthesia the interstitial fluid migrating in to the circulating compartment (Sankar et al., 2011), might also have led to haemo dilution and subsequent reduction in volume of packed red cells values (Kavechiya, 2010). The reason for the reduction in hepatic function parameters during anaesthetic period could be due to hepatic hypoperfusion with anaesthetic agents (Meierhenrich et al. 2009). Since liver and kidney functions will not be altered by isoflurane anaesthesia (Sen and Kilic, 2018), the values like creatinine and blood urea nitrogen were within the normal range in all the animals under study.

\section{Conclusion}

The quality of sedation, induction, maintenance and recovery from general anaesthesia were good without any complications. The present anaesthetic protocol was found be safe in the geriatric 
animals with regard to haematological and serum biochemical parameters as the variations remained within the normal acceptable range.

\section{Acknowledgement}

The authors are thankful to the Dean, College of Veterinary and Animal Sciences, Mannuthy for providing all the necessary facilities to conduct the research work and giving permission to publish this work.

\section{References}

Baetge, C.L. and Matthews, N.S. 2012. Anesthesia and analgesia for geriatric veterinary patients. Vet. Clin. N. Am. Small Anim. Pract. 42: 643-653.

Brand, J.M., Frohn, C., Luhm, J., Kirchner, H. and Schmucker, P. 2003. Early alterations in the number of circulating lymphocyte subpopulations and enhanced proinflammatory immune response during opioid-based general anesthesia. Shock. 20: 213-217.

Joubert, K.E. 2007. Pre-anaesthetic screening of geriatric dogs. J. S. Afri. Vet. Ass. 78: 31-35.

Kavechiya, V.P. 2010. Studies on balanced anaesthesia using butorphanolacepromazine-glycopyrrolate (BAG) as preanaesthetic to ketamine-diazepam, ketamine-midazolam, propofol and isoflurane maintenance in canines. M.V.Sc. thesis, Anand Agricultural University, Anand, 137p.

Meierhenrich, R., Wagner, F., Schutz, W., Rockemann, M., Steffen, P., Senftleben, thoracic epidural anesthesia on hepatic blood flow in patients under general anesthesia. Anaesth. Analg. 108: 13311337.

Ramankutty, S. 2008. Clinical evaluation of propofol-isoflurane anaesthesia with xylazine premedication in dogs. M.V.Sc. thesis, Kerala Agricultural University, Thrissur, 137p.

Sankar, P., William, B.J., Rao, G.D., Prathaban, S., Kumar, R.S. and Leela, V. 2011. Cardiopulmonary and haematobiochemical alterations during ketamine or propofol anaesthesia in acepromazine-xylazine premedicated horses. Indian J. Vet. Surg. 32: 23-26.

Sen, Z.B. and Kilic, N. 2018. General anesthesia in geriatric dogs with propofol-isoflurane, propofol-sevoflurane, alphaxaloneisoflurane, alphaxalone-sevoflurane and their comparison of biochemical, hemodynamic and cardiopulmonary effects. Acta. Sci. Vet. 46: 1-9.

Tomihari, M., Nishihara, A., Shimada, T., Yanagawa, M., Miyoshi, M., Miyahara, K. and Oishi, A. 2015. A comparison of the immunological effects of propofol and isoflurane for maintenance of anesthesia in healthy dogs. J. Vet. Med. Sci. 77: 1227-1233.

Zlateva, N. and Marinov, G. 2015. Effect of three anesthetic protocols on the haematological indices in cats during ovariohysterectomy. Med. Info. 2: 184193. 